\title{
A fluid model for a relay node in an ad hoc network: the case of heavy-tailed input
}

\author{
R. Bekker • M. Mandjes
}

Received: 4 September 2007 / Revised: 25 August 2008 / Published online: 7 November 2008 C) The Author(s) 2008. This article is published with open access at Springerlink.com

\begin{abstract}
Relay nodes in an ad hoc network can be modelled as fluid queues, in which the available service capacity is shared by the input and output. In this paper such a relay node is considered; jobs arrive according to a Poisson process and bring along a random amount of work. The total transmission capacity is fairly shared, meaning that, when $n$ jobs are present, each job transmits traffic into the queue at rate $1 /(n+1)$ while the queue is drained at the same rate of $1 /(n+1)$. Where previous studies mainly concentrated on the case of exponentially distributed job sizes, the present paper addresses regularly varying jobs. The focus lies on the tail asymptotics of the sojourn time $S$. Using sample-path arguments, it is proven that $\mathbb{P}\{S>x\}$ behaves roughly as the residual job size, i.e., if the job sizes are regularly varying of index $-v$, the tail of $S$ is regularly varying of index $1-v$. In addition, we address the tail
\end{abstract}

Part of the research was conducted while R. Bekker was affiliated to CWI. Hans van den Berg (Telecom ICT) is acknowledged for bringing this model under our attention.

R. Bekker $(\bowtie)$

Department of Mathematics, Vrije Universiteit, De Boelelaan 1081a,

1081 HV Amsterdam, The Netherlands

e-mail: rbekker@few.vu.nl

M. Mandjes

Korteweg-de Vries Institute for Mathematics, Plantage Muidergracht 24,

1018 TV Amsterdam, The Netherlands

M. Mandjes

CWI, Amsterdam, The Netherlands

M. Mandjes

EURANDOM, Eindhoven, The Netherlands

e-mail: M.R.H.mandjes@uva.nl 
asymptotics of other performance metrics, such as the workload in the queue, the flow transfer time and the queueing delay.

Keywords Queueing · Asymptotics · Regular variation · Ad hoc networks

\section{Introduction}

Ad hoc networks are self-configuring networks of mobile routers, connected by wireless links. They enable infrastructure-free communication: no fixed equipment is needed, but instead each client acts as a hub. When information needs to be transmitted across the network, it is sent from the sender to the receiver by relaying the packets along intermediate nodes. An excellent survey on ad hoc networks, with special emphasis on Quality-of-Service aspects, is Reddy et al. (2006).

On an abstract level one could model nodes in an ad hoc network as queues, see Van den Berg et al. (2006). Indeed, data packets arrive from source nodes and are served to be relayed to destination nodes. The complicating property is that the total transmission capacity is fixed and has to be shared between (i) source nodes sending information packets into the queue, and (ii) the service process at the queue of transmitting information packets towards 'successor nodes' (and eventually the destination client). Hence, the present model differs from ordinary queueing models by the fact that the total tranmission rate, which is commonly used completely for serving the queue, is now shared between the arrival and service processes.

To describe the sharing mechanism of the transmission rate, consider the situation that at some point in time $n$ stations send traffic through the same relay node. Then each 'sending node' is assigned an equal share $1 /(n+1)$ of the available medium capacity (which we may normalize to 1 ), which is the same fraction as is allocated to serve the queue. Note that the total input rate is thus $n /(n+1)$, so that the net rate of growth of the queue is $(n-1) /(n+1)$. We conclude that as soon as $n>1$, the node's input rate exceeds its output rate, and hence the excess traffic accumulates in the node's buffer; only when $n=0$ the queue drains (see also Mandjes and Roijers 2007 for an illustration of the model dynamics). Interestingly, this entails that relay nodes are prone to becoming bottlenecks. We remark that the queue is served on a First Come First Served (FCFS) basis.

The above assumption of equally sharing the transmission capacity is based on the mathematical description of an IEEE 802.11B protocol that is proposed and being implemented, see e.g. Reddy et al. (2006). However, one could think of other allocation policies, for instance, by increasing the transmission share of the relay node (see also Mandjes and Roijers 2007, Sect. 8). In that instance, the queueing delay will decrease, but that is at the expense of the time required to put the job into the buffer of the relay node. We refer to Roijers et al. (2008) for further details.

To study the relay node described above, we consider a flow-level model. Jobs arrive at the relay node, for instance, according to a Poisson process, and bring along a random amount of work, say i.i.d. (independent and identically distributed) samples from a distribution $B(\cdot)$. At the flow level, traffic then arrives as a fluid to the queue. 
There are several interesting performance measures to consider. In the first place, one may be interested in the time $F$ before a job is completely 'pulled out of the predecessor node', in that all traffic has reached the queue of the relay node. It then still takes some time, however, before the job has gone through the relay node: the sojourn time $S$ equals the sum of $F$ and the delay $D$ of the last particle of the flow. Previous work by Mandjes and Roijers (2007) focused on the case that $B(\cdot)$ corresponds to an exponential distribution, and it is a natural question whether other distributions are amenable for analysis as well.

In this paper we consider the relevant, and technically interesting, case of heavytailed jobs. If the network is used for file transfer purposes it is likely that traffic has heavy-tailed characteristics. This follows from extensive measurement studies showing that file sizes in the Internet commonly have heavy-tailed features, see e.g. Crovella and Bestavros (1996). More precisely, we assume that the jobs are i.i.d. samples from a regularly varying distribution of index - v, i.e., $\mathbb{P}\{B>x\}$ behaves roughly like $x^{-v}$, for some $v>0$; we write $B(\cdot) \in \mathcal{R}_{-v}$. For standard queueing models (i.e., models of $\mathrm{M} / \mathrm{G} / 1$ or $\mathrm{GI} / \mathrm{G} / 1$ type) with regularly varying input, a wealth of interesting contributions have appeared; early papers are for instance Cohen (1973); Pakes (1975). Generally speaking, under FCFS scheduling the sojourn time (just like the workload) is in $\mathcal{R}_{1-v}$ (that is, the tail is as heavy as a residual job size), whereas under processorsharing (Zwart and Boxma 2000) it is in $\mathcal{R}_{-v}$ (and hence the tail is essentially as heavy as that of the jobs themselves). As is clear from the model description we gave above, our relay-node has both PS and FCFS elements, and therefore it is an interesting fundamental question whether the sojourn time is in $\mathcal{R}_{1-v}$ or $\mathcal{R}_{-v}$ (or perhaps regularly varying of another index).

In our analysis, we rely on sample-path methods, comparable to those developed in Bekker et al. (2005). A lower bound is derived by identifying a most likely scenario, and computing its tail asymptotics. Then, in the upper bound we split the event of our interest into a number of sub-events, and show that among these, asymptotically, only the most likely scenario is relevant.

This paper is organized as follows. Section 2 details the model and presents some preliminaries. Section 3 describes the main results, which are proven in Sect. 4. Section 5 concludes the paper.

\section{Model and preliminaries}

In this section, we first give a description of the fluid-flow queueing system that is used to model a relay node in an ad hoc network. Second, we give some preliminary results that are mainly used in the proofs of our results.

\section{Model description}

Consider a queueing system at which flows arrive according to a Poisson process with rate $\lambda$. Each active flow brings along an amount of work; we assume that the service requirements are i.i.d. with distribution $B(\cdot)$ and mean $\beta<\infty$. The flow transmits traffic into the queue (according to a procedure detailed below) until it has sent out its 
full service requirement; then we say that the flow becomes inactive. Evidently, the mean amount of work generated per unit time is $\varrho:=\lambda \beta$.

The total transmission capacity is, without loss of generality, normalized to 1 . This transmission capacity is fairly shared between all nodes present. This means that when there are $N(u)$ active flows at time $u$, each active flow transmits traffic into the queue at rate $1 /(N(u)+1)$. The service rate of the queue then also equals $1 /(N(u)+1)$, implying that the queue is only drained when there are no active flows (and remains constant when there is one flow present).

In this paper, particularly in the proofs, we frequently use terminology of fluidtandem queues; quantities associated with traffic of active flows that is not yet in the queue are labelled class 1 , while quantities associated with traffic present in the queue are labelled class 2 . The total available transmission capacity for the active flows (that is, the input rate of the queue) during the time interval $[s, t]$ thus equals

$$
C_{1}(s, t):=\int_{s}^{t} \frac{N(u)}{N(u)+1} \mathrm{~d} u
$$

and the total service rate for the queue equals

$$
C_{2}(s, t):=\int_{s}^{t} \frac{1}{N(u)+1} \mathrm{~d} u
$$

Since the input into the buffer during the interval $[s, t]$ equals the total transmissions of active flows, we obtain the following useful representation of the class- 2 workload:

$$
V_{2}(t)=\sup _{s \leq t}\left\{C_{1}(s, t)-C_{2}(s, t)\right\}=\sup _{s \leq t}\left\{\int_{s}^{t} \frac{N(u)-1}{N(u)+1} \mathrm{~d} u\right\} .
$$

In this paper, we assume that the service requirement distribution is heavy-tailed. Let $B$ denote a generic service requirement, and let $B^{r}$ be a random variable distributed as the residual lifetime of $B$, i.e.,

$$
B^{r}(x):=\mathbb{P}\left\{B^{r}<x\right\}=\frac{1}{\beta} \int_{0}^{x}(1-B(y)) \mathrm{d} y .
$$

More specifically, we assume that the service requirement distribution is regularly varying of index $-v\left(\right.$ denoted as $B(\cdot) \in \mathcal{R}_{-v}$ ), i.e., $1-B(x) \sim L(x) x^{-v}, v>1$ (so that $\beta<\infty$ ), with $L(x)$ some slowly varying function. Here, and throughout the paper, we use the notation $f(x) \sim g(x)$ to indicate that $f(x) / g(x) \rightarrow 1$ as $x \rightarrow \infty$. (A function $L(\cdot)$ is called slowly varying if $L(\eta x) \sim L(x)$ for all $\eta>1$.) It follows from Karamata's Theorem, see Bingham et al. 1987 [Thm. 5.1.11], that $x \mathbb{P}\{B>x\} \sim(v-1) \beta \mathbb{P}\left\{B^{r}>x\right\}$, and thus $B^{r}(\cdot) \in \mathcal{R}_{1-v}$. 


\section{Preliminaries}

Denote by $B_{i}(s, t), i=1,2$, the amount of service received by class $i$ during the interval $[s, t]$. The amounts of service satisfy the following evident inequality

$$
B_{1}(s, t)+B_{2}(s, t) \leq t-s,
$$

with equality iff $V_{1}(u)+V_{2}(u)>0$ for all $u \in[s, t]$.

Similarly, define $A_{i}(s, t), i=1,2$, as the total input for class $i$ during the interval $[s, t]$. For the workloads the following obvious identity relation holds, for $i=1,2$ and $s<t$,

$$
V_{i}(t)=V_{i}(s)+A_{i}(s, t)-B_{i}(s, t) .
$$

Furthermore, using the fact that $A_{2}(s, t)=B_{1}(s, t)$, we have

$$
V_{2}(t)=V_{2}(s)+B_{1}(s, t)-B_{2}(s, t) .
$$

$A_{1}(s, t)$ is distributed as a Poisson number (with mean $\lambda(t-s)$ ) of i.i.d. service requirements, each with distribution $B(\cdot)$, and the class-1 workload obeys

$$
V_{1}(t)=\sup _{s \leq t}\left\{A_{1}(s, t)-\int_{s}^{t} \frac{N(u)}{N(u)+1} \mathrm{~d} u\right\} .
$$

We note that the above entails that the workload in the overall system can be directly related to the stationary workload in an M/G/1 queueing model. This can be seen as follows. In our tandem-queueing model, arriving flows are essentially served twice, see Van den Berg et al. (2006); flows that belong to the class-1 workload require two stages of service, while for work in the class-2 workload (that is, the queue) there is only a single stage of service left. This entails that the stability constraint of the model is $\varrho<\frac{1}{2}$. Furthermore, it holds that the total service capacity (at a constant rate of 1 per unit time) is used as long as there is any work present, which entails that the system is work-conserving. According to Reich's formula (Reich 1958), the steady-state overall-workload representation therefore reads

$$
2 V_{1}+V_{2}={ }_{\mathrm{d}} \sup _{t \geq 0}\left\{2 A_{1}(-t, 0)-t\right\}
$$

The distribution of $2 V_{1}+V_{2}$ thus equals the steady-state workload distribution of an $\mathrm{M} / \mathrm{G} / 1$ queue with generic service requirement $2 B$. Applying the well-known asymptotic result for the standard M/G/1 queue, see Cohen (1973); Pakes (1975), we directly obtain the asymptotic tail distribution of the overall workload:

Theorem 2.1 Assume that $\varrho<1 / 2$. Then, $B(\cdot) \in \mathcal{R}_{-v}$ iff $\mathbb{P}\left\{2 V_{1}+V_{2}<\cdot\right\} \in \mathcal{R}_{1-v}$, and then

$$
\mathbb{P}\left\{2 V_{1}+V_{2}>x\right\} \sim \frac{2 \varrho}{1-2 \varrho} \mathbb{P}\left\{B^{r}>\frac{x}{2}\right\} .
$$


Finally, we focus on the time required to serve an amount of work in the queue. Let time 0 be an arbitrary instant at which a flow becomes active and let

$$
W_{0}:=\arg \inf _{t \geq 0}\left\{B_{2}(0, t)=V_{2}(0)\right\}
$$

be the time required to serve the amount of work present in the queue at flow initiation. Since active flows initiate their transmission immediately upon arrival, $W_{0}$ also corresponds to the epoch at which the first packet (to be interpreted as infinitesimally small fluid particle) of the flow leaves the buffer. In queueing terminology, this quantity is frequently referred to as the waiting time. Because the total transmission capacity is used during $\left[0, W_{0}\right]$, we have the following identity:

$$
W_{0}=V_{2}(0)+B_{1}\left(0, W_{0}\right) .
$$

We note that we interchangeably use $W$ and $W_{0}$ to denote such a generic waiting time.

\section{Results}

In this section, we present the main results of the paper. In particular, we give exact asymptotics for the steady-state workload, flow-transfer delay, queueing delay, and sojourn time. For the former two quantities this section also provides the proofs; the proofs for the latter two quantities (which are considerably more involved) are given in the next section. For each quantity, we also provide the underlying heuristics; these turn out to be extremely useful in understanding the model's properties (and play an important role in the proofs of Sect. 4).

\subsection{Steady-state workload}

We first consider the steady-state class- 2 workload distribution $V_{2}$. For this quantity we can rely on the main result of Bekker et al. (2005) giving the workload asymptotics of (in the terminology of Bekker et al. 2005) the streaming traffic, sharing bandwidth with a second class of elastic flows according to the PS discipline. The result indicates that the tail of the steady-state workload is as heavy as that of $B^{r}$, i.e., regularly varying of index $1-v$.

Theorem 3.1 If $B(\cdot) \in \mathcal{R}_{-v}$ and $0<\varrho<\frac{1}{2}$, then

$$
\mathbb{P}\left\{V_{2}>x\right\} \sim \frac{2 \varrho}{1-2 \varrho} \mathbb{P}\left\{B^{r}>\frac{1-\varrho}{2 \varrho} x\right\} .
$$

Proof Using (1), we may rewrite the workload representation as

$$
\begin{aligned}
V_{2}(t) & =\sup _{s \leq t}\left\{t-s-\int_{s}^{t} \frac{2}{N(u)+1} \mathrm{~d} u\right\} \\
& =2 \sup _{s \leq t}\left\{\frac{1}{2}(t-s)-\int_{s}^{t} \frac{1}{N(u)+1} \mathrm{~d} u\right\}=: 2 V^{\star}(t) .
\end{aligned}
$$


We note that $V^{\star}(t)$ equals the workload of the streaming class in case $K=1$ and $r=1 / 2$, using the terminology of the workload representation in Bekker et al. (2005). In that case, the condition $K r<1-\varrho<(K+1) r$ translates into $0<\varrho<1 / 2$. Now, using (7) and applying Bekker et al. (2005) (Thm. 4.1) with $K=1$ and $r=1 / 2$ gives the result.

\section{Heuristic arguments}

The heuristics behind the workload asymptotics are as follows. Consider the workload at an arbitrary instant, say, at time 0 . The most likely way for $V_{2}(0)$ to become large is the arrival of one exceptionally large job (also referred to as tagged job) of size $B_{\text {tag }}$ before time $-\tilde{t}_{1}$ (which is defined below). Suppose that this job arrives at time $-y$. For any value of $y \geq \tilde{t}_{1}$ one can determine the minimal size of the tagged job to make sure that $V_{2}(0)>x$.

We first observe that $y$ cannot be smaller than

$$
\tilde{t}_{1}:=\frac{x}{\varrho} .
$$

This can be seen as follows. The amount of work stored in the queue by all jobs except for the tagged one is close to its average amount of work generated, i.e., roughly $\varrho y$ arrives to the queue due to the other job arrivals during the interval $[-y, 0]$. Assuming that the tagged job is still transmitting into the buffer at time 0 (otherwise $V_{2}(0)$ would even be smaller), it follows from the PS discipline that the tagged job brings along as much work as is served by the queue; the buffer content at time 0 is then about $\varrho y$. For $V_{2}(0)>x$ it is thus required that $y>\tilde{t}_{1}$.

Suppose that the tagged job has size $B_{\text {tag. }}$. Over the duration of the transmission of the tagged job, it equally shares the remaining capacity with the queue at a rate $(1-\varrho) / 2$. It then takes $2 B_{\operatorname{tag}} /(1-\varrho)$ time to fully put the tagged job into the buffer, i.e., at time $-y+2 B_{\mathrm{tag}} /(1-\varrho)$ the tagged job has transmitted its full service request. Note that for the minimal size of $B_{\text {tag }}$ it holds that this time should be before time 0 . When the tagged job has fully arrived at the queue, the buffer drains at a rate $1-2 \varrho$. Thus, the buffer content at time 0 is

$$
V_{2}(0)=\frac{2 B_{\mathrm{tag}}}{1-\varrho} \varrho-\left(y-\frac{2 B_{\mathrm{tag}}}{1-\varrho}\right)(1-2 \varrho)=2 B_{\mathrm{tag}}-(1-2 \varrho) y .
$$

To make sure that $V_{2}(0)>x$, we have

$$
B_{\operatorname{tag}}>\frac{x}{2}+\frac{1-2 \varrho}{2} y .
$$

Integrating with respect to $y$ (and neglecting the asymptotically small probability of having two or more large job arrivals), it follows that the probability of a large workload roughly equals

$$
\int_{y=\tilde{t}_{1}}^{\infty} \lambda \mathbb{P}\left\{B_{\mathrm{tag}}>\frac{x}{2}+\frac{1-2 \varrho}{2} y\right\} \mathrm{d} y .
$$


After the change of variable $z=y-\tilde{t}_{1}$, dividing and multiplying with $\beta$, and using (2), we obtain the desired expression.

\subsection{Flow transfer delay}

Here we consider the time $F$ it takes for an arbitrary arriving flow to transmit its traffic into the buffer. Since the available transmission capacity at time $u$ for each individual active flow equals $1 /(N(u)+1)$ it trivially follows that the flow transfer delay equals the sojourn time of a non-permanent customer in a M/G/1 PS queue with one permanent customer. Applying Guillemin et al. (2003), Thm. 3, it follows directly that the flow transfer delay is regularly varying of index $-v$ :

Proposition 3.2 If $B(\cdot) \in \mathcal{R}_{-v}$ and $0<\varrho<1$, then

$$
\mathbb{P}\{F>x\} \sim \mathbb{P}\left\{B>\frac{1-\varrho}{2} x\right\} .
$$

Proof The result follows directly from Guillemin et al. (2003), Thm. 3, with the identification $\gamma^{f}:=(1-\varrho) / 2$, see also Bekker et al. (2005), Prop. 3.1.

\section{Heuristic arguments}

Clearly, the heuristics behind the flow-transfer delay asymptotics are the same as the heuristics for the asymptotic sojourn time in a M/G/1 PS queue with one permanent customer. That is, a large flow-transfer delay is due to a large service requirement of the flow itself. The ratio $(1-\varrho) / 2$ is simply the average service rate received by the large flow; over the duration of the large flow, the other flows transmission rate roughly equals their average input rate $\varrho$. The remaining capacity of $1-\varrho$ is equally shared between the large flow and the relay node (i.e., the buffer).

\subsection{Sojourn time and queueing delay}

In this part, we consider the queueing delay $D$ and sojourn time $S=F+D$ of an arbitrary arriving flow. The queueing delay is here defined as the time it takes the last packet (recall that a packet is to be interpreted as an infinitesimally small fluid particle) of the flow to go through the queue. The sojourn time is the time between the arrival of a flow until the last packet leaves the buffer.

In fact, the queueing delay and sojourn time are asymptotically equivalent, as presented in the following theorem:

Theorem 3.3 Define $\phi_{\max }:=\max \{1-\varrho,(1+\varrho) / 2\}$ and $\phi_{\min }:=\min \{1-\varrho,(1+$ $\varrho) / 2\}$. If $B(\cdot) \in \mathcal{R}_{-v}$ and $0<\varrho<\frac{1}{2}\left(\varrho \neq \frac{1}{3}\right)$, then

$$
\begin{gathered}
\mathbb{P}\{S>x\} \sim \mathbb{P}\{D>x\} \sim \frac{2 \varrho}{1-2 \varrho} \mathbb{P}\left\{B^{r}>\frac{(1-\varrho)^{2}}{2 \varrho} x\right\} \\
+\frac{2 \varrho}{|1-3 \varrho|} \mathbb{P}\left\{\phi_{\min } \frac{(1-\varrho)}{2 \varrho} x<B^{r}<\phi_{\max } \frac{(1-\varrho)}{2 \varrho} x\right\}
\end{gathered}
$$


Proof For any flow, the waiting time is evidently less than its sojourn time. Lower and upper bounds for the sojourn time $S$ that asymptotically coincide are then given by Propositions 4.2 and 4.3, respectively, providing the asymptotic tail of $S$.

Since the delay of a flow is bounded by its sojourn time, Proposition 4.3 also gives an asymptotic upper bound for the tail of $D$. For the lower bound, write

$$
\begin{aligned}
\mathbb{P}\{D>x\} & \geq \mathbb{P}\{S>(1+\epsilon) x ; F<\epsilon x\} \\
& \geq \mathbb{P}\{S>(1+\epsilon) x\}-\mathbb{P}\{F>\epsilon x\}
\end{aligned}
$$

Using Proposition 3.2 and the fact that $B(\cdot) \in \mathcal{R}_{-v}$ it follows that

$$
\mathbb{P}\{F>\epsilon x\}=o\left(\mathbb{P}\left\{B^{r}>x\right\}\right)
$$

The lower bound of $D$ now follows directly by letting $\epsilon \downarrow 0$ and using the fact that $S \in \mathcal{R}_{1-v}$, completing the proof.

Corollary 3.4 If $\mathbb{P}\{B>x\}=L(x) x^{-v}$ for some slowly varying $L(\cdot)$ and $0<\varrho<\frac{1}{2}$, then $S$ and $D \in \mathcal{R}_{1-v}$, and, in particular,

$$
\mathbb{P}\{S>x\} \sim \mathbb{P}\{D>x\} \sim \frac{1}{(v-1) \beta} L(x) x^{1-v} \cdot\left(\xi_{1}(\varrho)+\xi_{2}(\varrho)\right)
$$

where

$$
\begin{aligned}
& \xi_{1}(\varrho):=\frac{2 \varrho}{1-2 \varrho}\left(\frac{2 \varrho}{(1-\varrho)^{2}}\right)^{\nu-1} ; \\
& \xi_{2}(\varrho):=\frac{2 \varrho}{|1-3 \varrho|}\left(\left(\frac{2 \varrho}{\phi_{\min }(1-\varrho)}\right)^{\nu-1}-\left(\frac{2 \varrho}{\phi_{\max }(1-\varrho)}\right)^{\nu-1}\right) .
\end{aligned}
$$

Remark 3.5 Notice that the value $\varrho=\frac{1}{3}$ plays a special role in Corollary 3.4 (and Theorem 3.3). First observe that $\varrho<\frac{1}{3}$ holds iff $1-\varrho>(1+\varrho) / 2$; for $\varrho=\frac{1}{3}$ we have that $\phi_{\min }$ and $\phi_{\max }$ are equal. Elementary calculus shows that this entails that $\xi_{2}(\varrho)$ can be alternatively written as

$$
\xi_{2}(\varrho)=\frac{2 \varrho}{1-3 \varrho}\left(\left(\frac{4 \varrho}{1-\varrho^{2}}\right)^{\nu-1}-\left(\frac{2 \varrho}{(1-\varrho)^{2}}\right)^{\nu-1}\right)
$$

L'Hôptital's rule yields that

$$
\xi_{2}\left(\frac{1}{3}\right)=\frac{3}{4}(v-1)\left(\frac{3}{2}\right)^{v-2}
$$


so that for $\varrho=\frac{1}{3}$

$\mathbb{P}\{S>x\} \sim \mathbb{P}\{D>x\} \sim \frac{1}{(v-1) \beta} L(x) x^{1-v} \cdot\left(2\left(\frac{3}{2}\right)^{v-1}+\frac{3}{4}(v-1)\left(\frac{3}{2}\right)^{v-2}\right)$.

Remark 3.6 In Mandjes and Roijers (2007), the (virtual) queueing delay is defined as the delay experienced by a fluid particle arriving at the buffer at a random point in time. Using PASTA, it follows that the buffer content and number of flows present at the Poisson instants of flow arrivals are equal to these quantatities at arbitrary instants (time averages). Hence, the above definition of the queueing delay distribution is identical to the 'waiting time' distribution of the present paper and thus has the same asymptotic behavior.

\section{Heuristic arguments}

The heuristics of the sojourn time and queueing delay are as follows. Consider the job that arrives at, say, time 0 . This job has an exceptionally long sojourn time if it sees upon arrival an exceptionally large workload, while the job itself is relatively small. This large workload is in turn due to a single exceptionally large job (to which we refer to as the tagged job) that arrived in the past at time, say, $-y$. Because the job itself is small, the flow transfer delay can be neglected compared to the queueing delay, yielding the same asymptotic behavior for the queueing delay, sojourn time, and also the waiting time. In the heuristics we henceforth focus on the waiting time $W_{0}$, i.e., the time required to serve $V_{2}(0)$.

For any value of $y>0$ one can determine the minimal size of the tagged job to make sure that work arriving at the queue at time 0 does not leave the system before time $x$.

We first observe that $y$ cannot be smaller than

$$
t_{0}:=\frac{(1-\varrho) x}{2 \varrho}
$$

This can be seen as follows. As long as the tagged job is in the system, the queue grows at a rate of roughly $\varrho$ (because the tagged job brings along as much work as is served by the queue, and hence all 'usual input', arriving at an average rate of $\varrho$, is stored in the queue). In other words, the buffer content at time 0 is about $\varrho y$. Now consider a fluid packet arriving at time 0 . To maximize the time before this packet leaves the queue, assume that the tagged job stays in the system; then the buffer drains at a rate $(1-\varrho) / 2$. This means that the queue is empty at time $2 \varrho y /(1-\varrho)$, which cannot be larger than $x$ if $y$ is smaller than $t_{0}$. Therefore we assume from now on $y \geq t_{0}$.

First we consider the situation that the tagged job has transmitted its full service requirement into the buffer at time 0 . The capacity used by all other customers to store traffic into the queue roughly equals the amount of work generated, which is close to average. Hence, after time 0 , the buffer is drained at a rate of $1-\varrho$, implying that

$$
W_{0} \approx \frac{V_{2}(0)}{1-\varrho}
$$



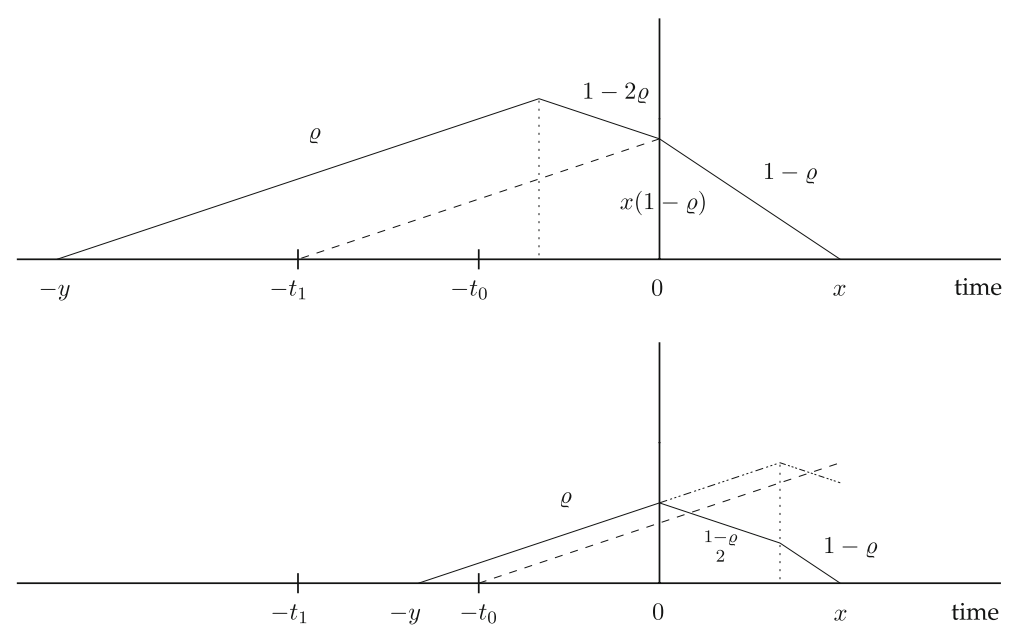

Fig. 1 Two realizations of a large waiting time $x$; before time 0 (i.e., left of the vertical axis) the total amount of work in the buffer is depicted, while after time 0 (i.e., right of the vertical axis) the amount of work in the buffer in front of the fluid particle arriving at time 0 is drawn

For $W_{0}>x$ it is thus sufficient that $V_{2}(0)>x(1-\varrho)$. Using the asymptotic results for $V_{2}(0)$ this directly provides the first term in the rhs of (8). We note that it follows directly from the heuristics of the workload asymptotics (see Sect. 3.1) that the most likely scenario for $V_{2}(0)>x(1-\varrho)$ to occur is the arrival of a large job at time $-y$, with

$$
y \geq t_{1}:=\frac{1-\varrho}{\varrho} x
$$

and with a service requirement

$$
B_{\operatorname{tag}}>\frac{1-\varrho}{2} x+\frac{1-2 \varrho}{2} y .
$$

The heuristic arguments for this scenario are also depicted in the first figure of Fig. 1.

Now consider the situation that the tagged job is still transmitting into the queue at time 0 . The tagged job can send traffic into the queue at a rate of about $(1-\varrho) / 2$. Supposing the tagged job has size $B_{\text {tag }}$, then it has been put into the buffer at time $-y+2 B_{\mathrm{tag}} /(1-\varrho)>0$; since there is roughly $\varrho y$ in the buffer at time 0 , the amount of work in front of the job arriving at time 0 left at that particular instant then equals

$$
\varrho y-\left(\frac{2 B_{\mathrm{tag}}}{1-\varrho}-y\right)\left(\frac{1-\varrho}{2}\right)=\varrho y-B_{\mathrm{tag}}+\frac{1}{2} y(1-\varrho) .
$$

In other words, the waiting time $W_{0}$ (or time to serve the amount of work at time 0 ) is

$$
-y+\frac{2 B_{\mathrm{tag}}}{1-\varrho}+\left(\varrho y-B_{\mathrm{tag}}+\frac{1}{2} y(1-\varrho)\right) /(1-\varrho)=\frac{B_{\mathrm{tag}}}{1-\varrho}+\frac{3 \varrho-1}{2(1-\varrho)} y
$$


this time is larger than $x$ if

$$
B_{\operatorname{tag}}>(1-\varrho) x+\frac{1}{2}(1-3 \varrho) y .
$$

See also the second figure of Fig. 1 for a graphical illustration of this scenario. It is readily verified that these $y$ should lie in the interval $\left[t_{0}, t_{1}\right]$.

Now, realizing that the probability that a job arriving in interval $\mathrm{d} y$ around $y$ is $\lambda \mathrm{d} y$, the probability of a long delay (and the probability of a long sojourn time) roughly equals

$$
\int_{t_{0}}^{t_{1}} \lambda \mathbb{P}\left\{B>(1-\varrho) x+\frac{1}{2}(1-3 \varrho) y\right\} \mathrm{d} y+\int_{t_{1}}^{\infty} \lambda \mathbb{P}\left\{B>\frac{1}{2}(1-\varrho) x+\frac{1-2 \varrho}{2} y\right\} \mathrm{d} y .
$$

After a change of variable, dividing and multiplying with $\beta$, and using the definition of the residual lifetime of $B$, we obtain the desired expression.

Remark 3.7 The special role of $\varrho=\frac{1}{3}$ may become apparent from Equation (9), indicating the value of the service requirement of the tagged flow arriving at time $-y$, $y \in\left[t_{0}, t_{1}\right]$, needed for the waiting time to be larger than $x$. Interestingly, the rhs of Equation (9) is decreasing (increasing) in $y$ for $\varrho>\frac{1}{3}\left(\varrho<\frac{1}{3}\right)$ and is independent of the arrival instant $-y$ in case $\varrho=\frac{1}{3}$. To understand the latter, suppose that the tagged job arrives at time $-(y+h)$ instead of at $-y$, with $h$ sufficiently small. The waiting time before the tagged job has been fully put into the buffer then obviously is $h$ time units smaller. Also, at that instant, the amount of work before the customer arriving at time 0 increases with $(\varrho+(1-\varrho) / 2) h$ (the amount of work at time 0 increases with $\varrho h$ and the second part follows from the fact the tagged job is $h$ time units less present after time 0 , during which the service rate roughly equals $(1-\varrho) / 2))$. The time required to serve this additional amount of work is $((\varrho+(1-\varrho) / 2) /(1-\varrho)) h=(1+\varrho) /(2(1-\varrho)) h$, which equals $h$ in case $\varrho=\frac{1}{3}$. Hence, this exactly compensates the reduction in waiting time before the tagged job has been put into the buffer.

\section{Proofs}

In this section we derive the asymptotics of the sojourn time and queueing delay, i.e., we prove the asymptotics of $\mathbb{P}\{S>x\}$ and $\mathbb{P}\{W>x\}$, as stated in Theorem 3.3. We consider the system at an arbitrary instant at which a flow becomes active, say at time 0 . As indicated earlier, we denote by $W \equiv W_{0}$ its "waiting time" (defined as the time until the first packet of the flow leaves the buffer). In Sect. 4.1, we obtain an asymptotic lower bound for $\mathbb{P}\left\{W_{0}>x\right\}$ and thus for $\mathbb{P}\left\{S_{0}>x\right\}$ (with $S_{0}$ denoting the sojourn time of our flow), while in Sect. 4.2 an asymptotic upper bound for $\mathbb{P}\left\{S_{0}>x\right\}$ that asymptotically coincides is derived. 


\subsection{Lower bound}

In this subsection, we derive an asymptotic lower bound for $\mathbb{P}\{S>x\}$ and $\mathbb{P}\{W>x\}$. First, we sketch two scenarios which enable us to show that these two scenarios provide sufficient sample-path conditions for the event $W_{0}>x$ to occur, see Lemma 4.1; it is instructive to compare these scenarios with those heuristically derived in the previous section. Next, we convert these sample-path inclusions into a probabilistic lower bound for $\mathbb{P}\left\{W_{0}>x\right\}$.

Let $\delta, \epsilon$ be sufficiently small positive constants and define two time instants $t_{0}<t_{1}$ as

$$
t_{0}:=\frac{1-\varrho+\delta+5 \epsilon}{2(\varrho-\delta)} x \quad \text { and } \quad t_{1}:=\frac{1-\varrho+\delta+3 \epsilon}{\varrho-\delta} x
$$

that is, $t_{0}$ is close to $x \cdot(1-\varrho) / 2 \varrho$, and $t_{1}$ is close to $x \cdot(1-\varrho) / \varrho$. Now, consider the following events:

1. Either $\exists y \in\left[t_{0}, t_{1}\right]$ such that at time $-y$ a tagged flow arrives with service requirement

$$
\begin{aligned}
B_{\mathrm{tag}} & \geq \frac{1-\varrho+\delta+5 \epsilon}{2(\varrho-\delta)} \frac{1+\varrho-\delta}{2} x+\frac{1-3(\varrho-\delta)}{2}\left(y-t_{0}\right)-\epsilon x \\
& =(1-\varrho+\delta+5 \epsilon) x+\frac{1-3(\varrho-\delta)}{2} y-\epsilon x,
\end{aligned}
$$

or $\exists y \geq t_{1}$ such that at time $-y$ a tagged flow arrives with service requirement

$$
\begin{aligned}
B_{\mathrm{tag}} & \geq \frac{1-\varrho+\delta}{2(\varrho-\delta)}(1-\varrho+\delta+3 \epsilon) x+\frac{1-2(\varrho-\delta)}{2}\left(y-t_{1}\right)+\epsilon x \\
& =\frac{1}{2}(1-\varrho+\delta+3 \epsilon) x+\frac{1-2(\varrho-\delta)}{2} y+\epsilon x .
\end{aligned}
$$

2. For the amount of arriving traffic it holds that

$$
A_{1}(-y, 0) \geq(\varrho-\delta) y-\epsilon x \quad \text { and } \quad A_{1}\left(0, W_{0}\right) \geq(\varrho-\delta) W_{0}-\epsilon x .
$$

3. The workload of class 1, except from the tagged flow, satisfies

$$
V_{1}^{-}(0) \leq \epsilon x \quad \text { and } \quad V_{1}^{-}\left(W_{0}\right) \leq \epsilon x
$$

The next lemma gives a sample-path relation between the scenarios given above and the event $W_{0}>x$.

Lemma 4.1 If either the events $\{(10),(12),(13)\}$ or $\{(11),(12),(13)\}$ occur simultaneously, then $W_{0}>x$. 
Proof Let us first consider the case that the events (10), (12), and (13) occur simultaneously. We now distinguish between two cases: (i) The large tagged flow-as defined through (10) - is still present at time $W_{0}$; and (ii) the tagged flow already left before time $W_{0}$.

Denote by $B_{1}^{\text {tag }}(s, t)$ and $B_{1}^{-}(s, t)$ the amount of service received by the tagged flow and by class 1 except for the tagged flow, respectively, during the interval $[s, t]$.

First consider case (i). Because the tagged flow is still present at time 0 , it follows from the PS discipline that $B_{1}^{\mathrm{tag}}(-y, 0)=B_{2}(-y, 0)$. Combining this with (5) and (4), yields

$$
\begin{aligned}
V_{2}(0) & =V_{2}(-y)+B_{1}^{-}(-y, 0)+B_{1}^{\mathrm{tag}}(-y, 0)-B_{2}(-y, 0) \\
& \geq A_{1}(-y, 0)-V_{1}^{-}(0) .
\end{aligned}
$$

From the PS discipline, it follows that $B_{1}^{\text {tag }}\left(0, W_{0}\right)=V_{2}(0)$ since the tagged flow is still present at time $W_{0}$. Combining this with (14) and using (6) and (4), we obtain

$$
\begin{aligned}
W_{0} & =V_{2}(0)+B_{1}^{\mathrm{tag}}\left(0, W_{0}\right)+B_{1}^{-}\left(0, W_{0}\right) \\
& \geq 2 A_{1}(-y, 0)+A_{1}\left(0, W_{0}\right)-V_{1}^{-}(0)-V_{1}^{-}\left(W_{0}\right) .
\end{aligned}
$$

Rewriting gives

$$
\begin{aligned}
(1-\varrho+\delta) W_{0} & \geq 2 A_{1}(-y, 0)+A_{1}\left(0, W_{0}\right)-(\varrho-\delta) W_{0}-V_{1}^{-}(0)-V_{1}^{-}\left(W_{0}\right) \\
& \stackrel{\text { (a) }}{\geq}(\varrho-\delta) y-5 \epsilon x \\
& \geq 2(\varrho-\delta) t_{0}-5 \epsilon x=(1-\varrho+\delta) x
\end{aligned}
$$

where the equality follows from the definition of $t_{0}$. Notice that in Inequality (a) we have used (12) and (13), whereas (10) does not need to be invoked in this case.

Next, consider case (ii). Applying (3) and (4), we have

$$
\begin{aligned}
B_{1}^{\mathrm{tag}}(-y, 0)+B_{2}(-y, 0) & \leq y-B_{1}^{-}(-y, 0) \\
& \leq y-A_{1}(-y, 0)+V_{1}^{-}(0)
\end{aligned}
$$

Because of the PS discipline, it holds that $B_{1}^{\mathrm{tag}}(-y, 0) \leq B_{2}(-y, 0)$ and, hence,

$$
B_{1}^{\mathrm{tag}}(-y, 0) \leq \frac{1}{2}\left(y-A_{1}(-y, 0)+V_{1}^{-}(0)\right)
$$

Observe that

$$
\begin{aligned}
B_{1}^{\mathrm{tag}}(-y, 0) & \leq(\varrho-\delta) y+\epsilon x+\frac{1-3(\varrho-\delta)}{2} y \\
& \leq(\varrho-\delta) t_{1}+\epsilon x+\frac{1-3(\varrho-\delta)}{2} y \\
& \leq(1-\varrho+\delta) x+4 \epsilon x+\frac{1-3(\varrho-\delta)}{2} y \leq B_{\mathrm{tag}}
\end{aligned}
$$


implying that the tagged flow is still active at time 0 . The lower bound (14) for $V_{2}(0)$ thus applies.

Also, because the tagged flow already left at time $W_{0}$, we have

$$
\begin{aligned}
B_{1}^{\mathrm{tag}}\left(0, W_{0}\right) & =B_{\mathrm{tag}}-B_{1}^{\mathrm{tag}}(-y, 0) \\
& \geq B_{\mathrm{tag}}-\frac{1}{2}\left(y-A_{1}(-y, 0)+V_{1}^{-}(0)\right) .
\end{aligned}
$$

Thus, using (4),

$$
\begin{aligned}
B_{1}\left(0, W_{0}\right) & =B_{1}^{\mathrm{tag}}\left(0, W_{0}\right)+B_{1}^{-}\left(0, W_{0}\right) \\
& \geq B_{\mathrm{tag}}-\frac{1}{2}\left(y-A_{1}(-y, 0)\right)+\frac{1}{2} V_{1}^{-}(0)+A_{1}\left(0, W_{0}\right)-V_{1}^{-}\left(W_{0}\right) .
\end{aligned}
$$

Hence, upon combining (6), (14), and (15), in addition to some rewriting, we obtain

$$
\begin{aligned}
(1-\varrho+\delta) W_{0} \geq & B_{\text {tag }}+\frac{3}{2} A_{1}(-y, 0)-\frac{1}{2} y+A_{1}\left(0, W_{0}\right) \\
& -(\varrho-\delta) W_{0}-\frac{1}{2} V_{1}^{-}(0)-V_{1}^{-}\left(W_{0}\right) \\
& \stackrel{\text { (b) }}{\geq} B_{\text {tag }}-\frac{1}{2}(1-3(\varrho-\delta)) y-4 \epsilon x \stackrel{\text { (c) }}{\geq}(1-\varrho+\delta) x,
\end{aligned}
$$

where step (b) follows from (12) and (13), and step (c) from (10). This completes the analysis of the first scenario.

We now turn to the case that the events (11), (12), and (13) occur simultaneously. Again, we distinguish between two cases: (i) The large tagged flow is still present at time 0 ; and (ii) the tagged flow already left before time 0 .

In case (i) the tagged flow is present at time 0 and the lower bound (14) for $V_{2}(0)$ thus applies again. Using (4), it follows that

$$
B_{1}\left(0, W_{0}\right) \geq B_{1}^{-}\left(0, W_{0}\right)=V_{1}^{-}(0)+A_{1}\left(0, W_{0}\right)-V_{1}^{-}\left(W_{0}\right) .
$$

Hence, combining (6), (14), and (16) gives

$$
\begin{aligned}
(1-\varrho+\delta) W_{0} & \geq A_{1}(-y, 0)+A_{1}\left(0, W_{0}\right)-(\varrho-\delta) W_{0}-V_{1}^{-}\left(W_{0}\right) \\
& \stackrel{(\mathrm{d})}{\geq}(\varrho-\delta) y-3 \epsilon x \\
& \geq(\varrho-\delta) t_{1}-3 \epsilon x=(1-\varrho+\delta) x
\end{aligned}
$$

where (d) follows from (12) and (13).

Next, consider case (ii). From (4) we obtain

$$
\begin{aligned}
B_{1}(-y, 0) & =B_{1}^{-}(-y, 0)+B_{1}^{\mathrm{tag}}(-y, 0) \\
& \geq A_{1}(-y, 0)-V_{1}^{-}(0)+B_{\mathrm{tag}} .
\end{aligned}
$$


Applying the above together with (5) and (3), yields

$$
\begin{aligned}
V_{2}(0) & \geq B_{1}(-y, 0)-B_{2}(-y, 0) \\
& \geq 2 B_{1}(-y, 0)-y \\
& \geq 2 B_{\mathrm{tag}}+2 A_{1}(-y, 0)-y-2 V_{1}^{-}(0)
\end{aligned}
$$

Now, it follows from (6), (16), and (17) that

$$
\begin{aligned}
(1-\varrho+\delta) W_{0} \geq & 2 B_{\mathrm{tag}}+2 A_{1}(-y, 0)-y+A_{1}\left(0, W_{0}\right) \\
& -(\varrho-\delta) W_{0}-V_{1}^{-}(0)-V_{1}^{-}\left(W_{0}\right) \\
& \stackrel{(\mathrm{e})}{\geq} 2 B_{\mathrm{tag}}-(1-2(\varrho-\delta)) y-5 \epsilon x \stackrel{\text { f })}{\geq}(1-\varrho+\delta) x ;
\end{aligned}
$$

here (e) follows from (12) and (13), and (f) from (11). This completes the sample-path analysis of the second scenario and the proof of the lemma.

In the next proposition, we convert the sample-path relation of Lemma 4.1 into a probabilistic lower bound for the tail distribution of $W_{0}$.

Proposition 4.2 (lower bound) If $B(\cdot) \in \mathcal{R}_{-v}$ and $0<\varrho<\frac{1}{2}\left(\varrho \neq \frac{1}{3}\right)$, then

$$
\begin{aligned}
& \mathbb{P}\{W>x\} \geq \frac{2 \varrho}{1-2 \varrho} \mathbb{P}\left\{B^{r}>\frac{(1-\varrho)^{2}}{2 \varrho} x\right\}(1+o(1)) \\
& +\frac{2 \varrho}{|1-3 \varrho|} \mathbb{P}\left\{\phi_{\min } \frac{1-\varrho}{2 \varrho} x<B^{r}<\phi_{\max } \frac{1-\varrho}{2 \varrho} x\right\}(1+o(1)), \quad x \rightarrow \infty
\end{aligned}
$$

Proof For notational convenience, we define

$$
\begin{aligned}
& g_{1}(\delta, \epsilon):=\frac{1-\varrho+\delta+5 \epsilon}{2(\varrho-\delta)} \frac{1+\varrho-\delta}{2}-\epsilon \\
& g_{2}(\delta, \epsilon):=\frac{1-\varrho+\delta}{2(\varrho-\delta)}(1-\varrho+\delta+3 \epsilon)+\epsilon
\end{aligned}
$$

To bound the two probabilities of (13) from below, we apply the M/G/1 PS model with 2 permanent customers and denote the workload at time $t$ in the latter model by $V_{\text {perm }}(t)$. We thus have that $V_{1}^{-}(t) \leq V_{\text {perm }}(t)$.

Using Lemma 4.1 and the observations above, we have

$$
\begin{gathered}
\mathbb{P}\left\{W_{0}>x\right\} \geq \mathbb{P}\left\{\exists y \in\left[t_{0}, t_{1}\right]: B_{\mathrm{tag}}>g_{1}(\delta, \epsilon) x\right. \\
\left.+\frac{1-3(\varrho-\delta)}{2}\left(y-t_{0}\right) ;(12) ;(13)\right\} \\
+\mathbb{P}\left\{\exists y \geq t_{1}: B_{\mathrm{tag}}>g_{2}(\delta, \epsilon) x+\frac{1-2(\varrho-\delta)}{2}\left(y-t_{1}\right) ;(12) ;(13)\right\}
\end{gathered}
$$




$$
\begin{aligned}
\geq & \left(\mathbb{P}\left\{\exists y \in\left[t_{0}, t_{1}\right]: B_{\text {tag }}>g_{1}(\delta, \epsilon) x+\frac{1-3(\varrho-\delta)}{2}\left(y-t_{0}\right)\right\}\right. \\
& \left.+\mathbb{P}\left\{\exists y \geq t_{1}: B_{\text {tag }}>g_{2}(\delta, \epsilon) x+\frac{1-2(\varrho-\delta)}{2}\left(y-t_{1}\right)\right\}\right) \\
& \times \mathbb{P}\left\{(12) ; V_{\text {perm }}(0) \leq \epsilon x ; V_{\text {perm }}\left(W_{0}\right) \leq \epsilon x\right\} .
\end{aligned}
$$

We now treat the three probabilities on the right-hand side of (18) separately. Starting with the second probability, we obtain, by integrating with respect to $y$, using the shorthand notation $\bar{g}(\varrho, \delta):=\frac{1}{2}-(\varrho-\delta)$,

$$
\begin{aligned}
& \mathbb{P}\left\{\exists y \geq t_{1}: B_{\mathrm{tag}}>g_{2}(\delta, \epsilon) x+\frac{1-2(\varrho-\delta)}{2}\left(y-t_{1}\right)\right\} \\
& \geq \int_{0}^{\infty} \lambda \mathbb{P}\left\{B_{\mathrm{tag}}>g_{2}(\delta, \epsilon) x+\bar{g}(\varrho, \delta) y\right\} \mathrm{d} y \\
& \quad-\int_{0}^{\infty} \int_{y}^{\infty} \lambda^{2} \mathbb{P}\left\{B_{\mathrm{tag}}>g_{2}(\delta, \epsilon) x+\bar{g}(\varrho, \delta) y ; B_{-z}>g_{2}(\delta, \epsilon) x+\bar{g}(\varrho, \delta) z\right\} \mathrm{d} z \mathrm{~d} y \\
& \sim \frac{2 \varrho}{1-2(\varrho-\delta)} \mathbb{P}\left\{B^{r}>g_{2}(\delta, \epsilon) x\right\}(1+o(1)),
\end{aligned}
$$

where $B_{-z}$ denotes the service requirement of a second large customer arriving at time $-z$ (see e.g. Boxma et al. 2003 for details on the asymptotically small probability of having two or more large flow arrivals).

The first probability on the right-hand side of (18) can be treated similarly. Neglecting the asymptotically small probability of two or more large customer arrivals again, we have

$$
\begin{aligned}
& \mathbb{P}\left\{\exists y \in\left[t_{0}, t_{1}\right]: B_{\mathrm{tag}}>g_{1}(\delta, \epsilon) x+\frac{1-3(\varrho-\delta)}{2}\left(y-t_{0}\right)\right\} \\
& =\int_{0}^{\infty} \lambda \mathbb{P}\left\{B_{\mathrm{tag}}>g_{1}(\delta, \epsilon) x+\frac{1-3(\varrho-\delta)}{2} y\right\} \mathrm{d} y \\
& \quad-\int_{t_{1}-t_{0}}^{\infty} \lambda \mathbb{P}\left\{B_{\mathrm{tag}}>g_{1}(\delta, \epsilon) x+\frac{1-3(\varrho-\delta)}{2} y\right\} \mathrm{d} y+o\left(\mathbb{P}\left\{B^{r}>x\right\}\right) \\
& \sim \frac{2 \varrho}{1-3(\varrho-\delta)}\left(\mathbb{P}\left\{B^{r}>g_{1}(\delta, \epsilon) x\right\}-\mathbb{P}\left\{B^{r}>g_{2}(\delta, \epsilon) x\right\}\right)(1+o(1)),
\end{aligned}
$$

where we used that $g_{2}(\delta, \epsilon) x=g_{1}(\delta, \epsilon) x+\left(t_{1}-t_{0}\right)(1-3(\varrho-\delta)) / 2$ in the final step.

For the third probability on the rhs of (18), we note that $A_{1}(-y, 0), A_{1}\left(0, W_{0}\right)$, $V_{\text {perm }}(0)$, and $V_{\text {perm }}\left(W_{0}\right)$ are not independent. We therefore write 


$$
\begin{aligned}
& \mathbb{P}\left\{(12) ; V_{\text {perm }}(0) \leq \epsilon x ; V_{\text {perm }}\left(W_{0}\right) \leq \epsilon x\right\} \\
& \geq \mathbb{P}\left\{A_{1}(-y, 0) \geq(\varrho-\delta) y-\epsilon x\right\} \times \mathbb{P}\left\{A_{1}\left(0, W_{0}\right) \geq(\varrho-\delta) W_{0}-\epsilon x\right\} \\
& \quad-\mathbb{P}\left\{V_{\text {perm }}(0)>\epsilon x\right\}-\mathbb{P}\left\{V_{\text {perm }}\left(W_{0}\right)>\epsilon x\right\} .
\end{aligned}
$$

Due to the (weak) law of large numbers we have that $\mathbb{P}\left\{A_{1}(-y, 0) \geq(\varrho-\delta) y-\epsilon x\right\}$ converges to 1 as $x \rightarrow \infty$; similarly $\mathbb{P}\left\{A_{1}\left(0, W_{0}\right) \geq(\varrho-\delta) W_{0}-\epsilon x\right\} \rightarrow 1$ as $x \rightarrow$ $\infty$. Since $V_{\text {perm }}(0)$ and $V_{\text {perm }}\left(W_{0}\right)$ have proper (that is, non-defective) distribution functions, it also holds that

$$
\lim _{x \rightarrow \infty} \mathbb{P}\left\{V_{\text {perm }}(0)>\epsilon x\right\}=0, \text { and } \lim _{x \rightarrow \infty} \mathbb{P}\left\{V_{\text {perm }}\left(W_{0}\right)>\epsilon x\right\}=0 .
$$

Finally, combining the above and using the fact that $B^{r}(\cdot) \in \mathcal{R}_{1-v}$, we have, as $x \rightarrow \infty$,

$$
\begin{aligned}
\mathbb{P}\{W>x\} \gtrsim & \frac{2 \varrho}{1-2(\varrho-\delta)} \mathbb{P}\left\{B^{r}>g_{2}(\delta, \epsilon) x\right\} \\
& +\frac{2 \varrho}{1-3(\varrho-\delta)}\left(\mathbb{P}\left\{B^{r}>g_{1}(\delta, \epsilon) x\right\}-\mathbb{P}\left\{B^{r}>g_{2}(\delta, \epsilon) x\right\}\right) \\
\rightarrow & \frac{2 \varrho}{1-2 \varrho} \mathbb{P}\left\{B^{r}>g_{2}(0,0) x\right\} \\
& +\frac{2 \varrho}{1-3 \varrho}\left(\mathbb{P}\left\{B^{r}>g_{1}(0,0) x\right\}-\mathbb{P}\left\{B^{r}>g_{2}(0,0) x\right\}\right), \delta, \epsilon \downarrow 0 ;
\end{aligned}
$$

here $f(x) \gtrsim g(x), x \rightarrow \infty$, indicates that $\liminf _{x \rightarrow \infty} f(x) / g(x) \geq 1$.

The proof is now completed by distinguishing between the cases $0<\varrho<\frac{1}{3}$ and $\frac{1}{3}<\varrho<\frac{1}{2}$.

\subsection{Upper bound}

In this subsection, we let time 0 correspond to an arbitrary flow arrival, and derive an asymptotic upper bound for $\mathbb{P}\left\{S_{0}>x\right\}$. In the proofs, we use a representation of the sojourn time $S_{0}$ that is similar to the waiting time representation (6). Let $F_{0}$ correspond to the flow transfer delay of the flow that arrives at time 0 and note that the events $S_{0} \geq x$ and $B_{2}\left(F_{0}, x\right) \leq V_{2}\left(F_{0}\right)$ are equivalent. Since the total service capacity is then used during $\left(F_{0}, x\right)$, the latter event can be rewritten as

$$
x-F_{0} \leq V_{2}\left(F_{0}\right)+B_{1}\left(F_{0}, x\right) .
$$

Moreover, we show in Proposition 4.3 that the most likely way for the sojourn time to become large is due to the arrival of a large tagged flow while the actual flow itself is small. It may be seen from Proposition 3.2 that the flow transfer delay $F_{0}$ of the 'small' flow is 'small' as well. Thus, assuming that $S_{0}>x$ and $F_{0} \leq \epsilon x$, with $\epsilon>0$ 
sufficiently small, we obtain the following relation:

$$
x(1-2 \epsilon) \leq V_{2}(0)+B_{1}(0, x) .
$$

This inequality relation will be the starting point for most of the sample-path relations in the proofs below.

Before turning to the proof of the asymptotic upper bound for $\mathbb{P}\left\{S_{0}>x\right\}$ (i.e. Proposition 4.3), we first introduce some notation that will be used throughout the section. Let $N_{b}(s, t)$ be the number of flows arriving in the interval $[s, t]$ with a service requirement satisfying $b$. In particular, we are interested in so-called 'large' flows that have a service requirement larger than $\kappa x$, for some $\kappa>0$ independent of $x$ (in which case we say that the service requirement is " $>\kappa x$ "). Also, for $t>0$, define

$$
W^{c}(0, t):=\sup _{0 \leq s \leq t}\left\{A_{1}(0, s)-c s\right\}
$$

and, for $u \leq v<0$,

$$
W^{c}(u, v):=\sup _{u \leq s \leq v}\left\{A_{1}(s, v)-c(v-s)\right\}
$$

In case the input process is modified such that only the flows with service requirements of at most $\kappa x$ are admitted, we add a subscript " $\leq \kappa x$ ", i.e., we write $W_{\leq \kappa x}^{c}(0, t)$ and $W_{\leq \kappa x}^{c}(u, v)$, respectively.

Again, let $\delta, \epsilon, \eta$ be sufficiently small positive constants. Similar to Sect. 4.1, define two time instants $0<s_{0}<s_{1}$ as

$$
s_{0}:=\frac{1-\varrho-\delta-2 \eta-10 \epsilon}{2(\varrho+\delta)} x \text { and } s_{1}:=\frac{1-\varrho-\delta-\eta-\frac{13}{2} \epsilon}{\varrho+\delta} x
$$

observe that $s_{0}$ is close to $t_{0}$, and $s_{1}$ to $t_{1}$. Let $s^{\star}:=\inf \left\{0 \leq t<s_{1}: V_{2}(-t)=0\right\}$ be the last epoch in $\left(-s_{1}, 0\right]$ that the system was empty, and let $s^{\star}=s_{1}$ in case $V_{2}(-t)>0$ for all $t \in\left(-s_{1}, 0\right]$. We have thus enforced that the total service capacity is used during the interval $\left[-s^{\star}, 0\right]$.

We are now settled for the proofs.

Proposition 4.3 (upper bound) If $B(\cdot) \in \mathcal{R}_{-v}$ and $0<\varrho<\frac{1}{2}\left(\varrho \neq \frac{1}{3}\right)$, then

$$
\begin{aligned}
& \mathbb{P}\{S>x\} \leq \frac{2 \varrho}{1-2 \varrho} \mathbb{P}\left\{B^{r}>\frac{(1-\varrho)^{2}}{2 \varrho} x\right\}(1+o(1)) \\
& +\frac{2 \varrho}{|1-3 \varrho|} \mathbb{P}\left\{\phi_{\min } \frac{1-\varrho}{2 \varrho} x<B^{r}<\phi_{\max } \frac{1-\varrho}{2 \varrho} x\right\}(1+o(1)), \quad x \rightarrow \infty .
\end{aligned}
$$

Proof First, we note that the premise for a large sojourn time to occur is that the arriving flow finds a large workload in the buffer, while the actual flow itself is small. 
Using Proposition 3.2, this implies that the flow transfer delay is small as well:

$$
\begin{aligned}
\mathbb{P}\left\{S_{0}>x\right\} & \leq \mathbb{P}\left\{S_{0}>x ; F_{0} \leq \epsilon x\right\}+\mathbb{P}\left\{F_{0}>\epsilon x\right\} \\
& =\mathbb{P}\left\{S_{0}>x ; F_{0} \leq \epsilon x\right\}+o\left(\mathbb{P}\left\{B^{r}>x\right\}\right) .
\end{aligned}
$$

We henceforth assume that $F_{0} \leq \epsilon x$.

There are in fact two 'most likely scenarios' for the event $S_{0}>x$ to occur; all the other scenarios are asymptotically negligible. To identify these most likely scenarios, decompose

$$
\begin{aligned}
\mathbb{P}\{ & \left.S_{0}>x ; F_{0} \leq \epsilon x\right\} \\
\leq & \mathbb{P}\left\{2 V_{1}\left(-s_{1}\right)+V_{2}\left(-s_{1}\right)>(1-\varrho-\delta-5 \epsilon) x+(1-2(\varrho+\delta)) s_{1}\right\} \\
+ & \mathbb{P}\left\{2 V_{1}\left(-s_{1}\right)+V_{2}\left(-s_{1}\right) \leq(1-\varrho-\delta-5 \epsilon) x\right. \\
& \left.+(1-2(\varrho+\delta)) s_{1} ; S_{0}>x ; F_{0} \leq \epsilon x\right\} .
\end{aligned}
$$

The first probability on the right-hand side of (23) contains the first most likely scenario. Note that the system is in steady state at time $-s_{1}$. Application of Theorem 2.1, in conjunction with the definition of $s_{1}$, then provides

$$
\begin{aligned}
& \mathbb{P}\left\{2 V_{1}\left(-s_{1}\right)+V_{2}\left(-s_{1}\right)>(1-\varrho-\delta-5 \epsilon) x+(1-2(\varrho+\delta)) s_{1}\right\} \\
& \sim \frac{2 \varrho}{1-2 \varrho} \mathbb{P}\left\{B^{r}>\frac{1-\varrho-\delta-\eta-\frac{13}{2} \epsilon}{2(\varrho+\delta)}(1-\varrho-\delta) x+\left(\eta+\frac{3}{2} \epsilon\right) x\right\} .
\end{aligned}
$$

We now turn to the second probability on the right-hand side of (23). Distinguishing between 0,1 , and 2 or more large-flow arrivals during $\left(-s_{1}, x\right]$ and the value of $2 V_{1}\left(-s_{1}\right)+V_{2}\left(-s_{1}\right)$ in case of 1 large-flow arrival during $\left(-s_{1}, x\right]$, we obtain

$$
\begin{gathered}
\mathbb{P}\left\{2 V_{1}\left(-s_{1}\right)+V_{2}\left(-s_{1}\right) \leq(1-\varrho-\delta-5 \epsilon) x\right. \\
\left.\quad+(1-2(\varrho+\delta)) s_{1} ; S_{0}>x ; F_{0} \leq \epsilon x\right\} \\
=P_{1}(x)+P_{2}(x)+P_{3}(x)+P_{4}(x),
\end{gathered}
$$

where

$$
\begin{aligned}
& P_{1}(x):=\mathbb{P}\left\{\begin{array}{c}
2 V_{1}\left(-s_{1}\right)+V_{2}\left(-s_{1}\right) \leq(1-\varrho-\delta-5 \epsilon) x+(1-2(\varrho+\delta)) s_{1} ; \\
N_{>\kappa x}\left(-s_{1}, x\right)=0 ; S_{0}>x ; F_{0} \leq \epsilon x
\end{array}\right\}, \\
& P_{2}(x):=\mathbb{P}\left\{\begin{array}{c}
2 V_{1}\left(-s_{1}\right)+V_{2}\left(-s_{1}\right) \leq \eta x ; \\
N_{>\kappa x}\left(-s_{1}, x\right)=1 ; S_{0}>x ; F_{0} \leq \epsilon x
\end{array}\right\}, \\
& P_{3}(x):=\mathbb{P}\left\{\begin{array}{c}
\eta x<2 V_{1}\left(-s_{1}\right)+V_{2}\left(-s_{1}\right) \leq(1-\varrho-\delta-5 \epsilon) x+(1-2(\varrho+\delta)) s_{1} ; \\
N_{>\kappa x}\left(-s_{1}, x\right)=1 ; S_{0}>x ; F_{0} \leq \epsilon x
\end{array}\right\}, \\
& P_{4}(x):=\mathbb{P}\left\{\begin{array}{c}
2 V_{1}\left(-s_{1}\right)+V_{2}\left(-s_{1}\right) \leq(1-\varrho-\delta-5 \epsilon) x+(1-2(\varrho+\delta)) s_{1} ; \\
N_{>\kappa x}\left(-s_{1}, x\right) \geq 2 ; S_{0}>x ; F_{0} \leq \epsilon x
\end{array}\right\} .
\end{aligned}
$$


The probability $P_{2}(x)$ contains the second most likely scenario, in that the probabilities $P_{1}(x), P_{3}(x)$, and $P_{4}(x)$ of the other scenarios are negligible relative to $P_{2}(x)$. The asymptotic behavior of $P_{2}(x)$ can be found in Lemma 4.4. Lemmas 4.7, 4.8, and 4.9 show that the terms $P_{1}(x), P_{3}(x)$, and $P_{4}(x)$, respectively, are negligible compared to $P_{2}(x)$ (and to the tail of the other most likely scenario).

To complete the proof, we let $\delta, \epsilon, \eta \downarrow 0$ (in (24) and (25)) and use the fact that $B^{r}(\cdot) \in \mathcal{R}_{1-v}$. The equivalence with (22) may be seen by distinguishing between $\varrho<\frac{1}{3}$ and $\varrho>\frac{1}{3}$ and some straightforward rewriting.

Lemma 4.4 For $\delta, \epsilon, \eta, \kappa>0$ sufficiently small $\left(\varrho+\delta \neq \frac{1}{3}\right)$ and $\varrho<\frac{1}{2}$, we have, as $x \rightarrow \infty$,

$$
\begin{aligned}
P_{2}(x) \lesssim & \frac{2 \varrho}{1-3(\varrho+\delta)}\left(\mathbb{P}\left\{B^{r}>\frac{1-\varrho-\delta-2 \eta-10 \epsilon}{2(\varrho+\delta)} \frac{1+\varrho+\delta}{2} x+\left(\eta+\frac{7}{2} \epsilon\right) x\right\}\right. \\
& \left.-\mathbb{P}\left\{B^{r}>\frac{1-\varrho-\delta-\eta-\frac{13}{2} \epsilon}{2(\varrho+\delta)}(1-\varrho-\delta) x\right\}\right) .
\end{aligned}
$$

Proof As in Sect. 4.1, denote the service requirement of the large tagged flow by $B_{\text {tag }}$ and let $-y, y \in\left[-x, s_{1}\right]$, be its arrival instant. We bound $P_{2}(x)$ by distinguishing between $y \geq s_{0}$ and $y<s_{0}$, and by the size of $B_{\text {tag }}$ in case $y \geq s_{0}$ :

$$
P_{2}(x) \leq P_{21}(x)+P_{22}(x)+P_{23}(x),
$$

where

$$
\begin{aligned}
& P_{21}(x):=\mathbb{P}\left\{\begin{array}{c}
2 V_{1}\left(-s_{1}\right)+V_{2}\left(-s_{1}\right) \leq \eta x ; \\
N_{>\kappa x}\left(-s_{1},-s_{0}\right)=0 ; N_{>\kappa x}\left(-s_{0}, x\right)=1 ; S_{0}>x ; F_{0} \leq \epsilon x
\end{array}\right\} \\
& P_{22}(x):=\mathbb{P}\left\{\begin{array}{c}
2 V_{1}\left(-s_{1}\right)+V_{2}\left(-s_{1}\right) \leq \eta x ; \\
B_{\operatorname{tag}} \leq\left(1-\varrho-\delta-\eta-\frac{13}{2} \epsilon\right) x+\frac{1}{2}(1-3(\varrho+\delta)) y, \quad y \in\left[s_{0}, s_{1}\right] ; \\
N_{>\kappa x}^{-}\left(-s_{1}, x\right)=0 ; S_{0}>x ; F_{0} \leq \epsilon x
\end{array}\right\} \\
& P_{23}(x):=\mathbb{P}\left\{\exists y \in\left[s_{0}, s_{1}\right]: B_{\mathrm{tag}}>\left(1-\varrho-\delta-\eta-\frac{13}{2} \epsilon\right) x+\frac{1}{2}(1-3(\varrho+\delta)) y\right\} .
\end{aligned}
$$

The probabilities $P_{21}(x)$ and $P_{22}(x)$ can be bounded by Lemmas 4.5 and 4.6, respectively, and are thus negligible compared to the dominant scenarios. For $P_{23}(x)$ we obtain, by integrating with respect to $y$,

$$
\begin{aligned}
P_{23}(x)= & \int_{s_{0}}^{\infty} \lambda \mathbb{P}\left\{B_{\mathrm{tag}}>\left(1-\varrho-\delta-\eta-\frac{13}{2} \epsilon\right) x+\frac{1}{2}(1-3(\varrho+\delta)) y\right\} \mathrm{d} y \\
& -\int_{s_{1}}^{\infty} \lambda \mathbb{P}\left\{B_{\mathrm{tag}}>\left(1-\varrho-\delta-\eta-\frac{13}{2} \epsilon\right) x+\frac{1}{2}(1-3(\varrho+\delta)) y\right\} \mathrm{d} y
\end{aligned}
$$




$$
\begin{aligned}
= & \frac{2 \varrho}{1-3(\varrho+\delta)}\left(\mathbb{P}\left\{B^{r}>\frac{1-\varrho-\delta-2 \eta-10 \epsilon}{2(\varrho+\delta)} \frac{1}{2}(1+\varrho+\delta) x+\left(\eta+\frac{7}{2} \epsilon\right) x\right\}\right. \\
& \left.-\mathbb{P}\left\{B^{r}>\frac{1-\varrho-\delta-\eta-\frac{13}{2} \epsilon}{2(\varrho+\delta)}(1-\varrho-\delta) x\right\}\right),
\end{aligned}
$$

where the second equality follows from the definitions of $s_{0}$ and $s_{1}$. This completes the proof.

Lemma 4.5 For $\delta, \epsilon, \eta, \kappa>0$ sufficiently small and $\varrho<\frac{1}{2}$, we have

$$
P_{21}(x)=o\left(\mathbb{P}\left\{B^{r}>x\right\}\right) \quad \text { as } x \rightarrow \infty .
$$

Proof Recall that $-s^{\star}$ represents the last epoch before time 0 that the system was empty. In case $s^{\star} \geq s_{0}$, we obtain from (5), (3), and (4) that

$$
\begin{aligned}
V_{2}\left(-s_{0}\right) & =V_{2}\left(-s^{\star}\right)+B_{1}\left(-s^{\star},-s_{0}\right)-B_{2}\left(-s^{\star},-s_{0}\right) \\
& =V_{2}\left(-s^{\star}\right)+2 B_{1}\left(-s^{\star},-s_{0}\right)-\left(s^{\star}-s_{0}\right) \\
& =2 V_{1}\left(-s^{\star}\right)+V_{2}\left(-s^{\star}\right)+2\left(A_{1}\left(-s^{\star},-s_{0}\right)-\frac{1}{2}\left(s^{\star}-s_{0}\right)\right)-2 V_{1}\left(-s_{0}\right) .
\end{aligned}
$$

Hence,

$$
2 V_{1}\left(-s_{0}\right)+V_{2}\left(-s_{0}\right) \leq 2 V_{1}\left(-s_{1}\right)+V_{2}\left(-s_{1}\right)+2 W^{\frac{1}{2}}\left(-s_{1},-s_{0}\right) .
$$

Define $s^{\prime}:=\min \left\{s^{\star}, s_{0}\right\}$, such that the full service capacity is used during $\left[-s^{\prime}, 0\right]$. Due to the PS discipline, it holds that $B_{2}\left(-s^{\prime}, 0\right) \geq B_{1}^{\mathrm{tag}}\left(-s^{\prime}, 0\right)$. Combining the above with (5) and (4) gives

$$
\begin{aligned}
V_{2}(0)= & V_{2}\left(-s^{\prime}\right)+B_{1}^{-}\left(-s^{\prime}, 0\right)+B_{1}^{\operatorname{tag}}\left(-s^{\prime}, 0\right)-B_{2}\left(-s^{\prime}, 0\right) \\
\leq & V_{2}\left(-s^{\prime}\right)+V_{1}^{-}\left(-s^{\prime}\right)+A_{1}^{-}\left(-s^{\prime}, 0\right)-(\varrho+\delta) s^{\prime}+(\varrho+\delta) s^{\prime}-V_{1}^{-}(0) \\
\leq & 2 V_{1}\left(-s_{1}\right)+V_{2}\left(-s_{1}\right)+2 W^{\frac{1}{2}}\left(-s_{1},-s_{0}\right) \\
& +W_{-\operatorname{tag}}^{\varrho+\delta}\left(-s_{0}, 0\right)+(\varrho+\delta) s_{0}-V_{1}^{-}(0)
\end{aligned}
$$

where $W_{- \text {tag }}^{c}(u, v)$ corresponds to $W^{c}(u, v)$ with the modification that the large tagged flow is excluded from the arrival process $\left(W_{-\operatorname{tag}}^{c}(0, t)\right.$ is defined similarly).

By again applying the properties of the PS discipline we find that $B_{1}^{\operatorname{tag}}\left(F_{0}, x\right) \leq$ $V_{2}\left(F_{0}\right)$ in case $S_{0}>x$. Under the condition that $F_{0} \leq \epsilon x$, we have $B_{1}^{\text {tag }}(0, x) \leq$ $V_{2}(0)+\epsilon x$. Together with (4), this yields

$$
\begin{aligned}
B_{1}(0, x) & =B_{1}^{-}(0, x)+B_{1}^{\mathrm{tag}}(0, x) \\
& \leq V_{1}^{-}(0)+A_{1}^{-}(0, x)+V_{2}(0)+\epsilon x .
\end{aligned}
$$


Now, by combining (27) and (28), we have

$$
\begin{aligned}
V_{2}(0)+B_{1}(0, x) \leq & 2 V_{2}(0)+V_{1}^{-}(0)+A_{1}^{-}(0, x)-(\varrho+\delta) x+(\varrho+\delta) x+\epsilon x \\
\leq & 2\left(2 V_{1}\left(-s_{1}\right)+V_{2}\left(-s_{1}\right)+2 W^{\frac{1}{2}}\left(-s_{1},-s_{0}\right)+W_{-\operatorname{tag}}^{\varrho+\delta}\left(-s_{0}, 0\right)\right) \\
& +2(\varrho+\delta) s_{0}+W_{-\operatorname{tag}}^{\varrho+\delta}(0, x)+(\varrho+\delta+\epsilon) x .
\end{aligned}
$$

Using (19) to convert this sample-path relation into a probabilistic bound gives

$$
\begin{aligned}
& P_{21}(x) \\
& \leq \mathbb{P}\left\{\begin{array}{c}
2\left(2 V_{1}\left(-s_{1}\right)+V_{2}\left(-s_{1}\right)+2 W^{\frac{1}{2}}\left(-s_{1},-s_{0}\right)+W_{-\operatorname{tag}}^{\varrho+\delta}\left(-s_{0}, 0\right)\right) \\
+2(\varrho+\delta) s_{0}+W_{-\operatorname{tag}}^{\varrho+\delta}(0, x)+(\varrho+\delta+\epsilon) x \geq x(1-2 \epsilon) ; \\
2 V_{1}\left(-s_{1}\right)+V_{2}\left(-s_{1}\right) \leq \eta x ; N_{>\kappa x}\left(-s_{1},-s_{0}\right)=0 ; N_{>\kappa x}\left(-s_{0}, x\right)=1
\end{array}\right\} \\
& \leq \mathbb{P}\left\{\begin{array}{c|c}
4 W^{\frac{1}{2}}\left(-s_{1},-s_{0}\right)+2 W_{-\operatorname{tag}}^{\varrho+\delta}\left(-s_{0}, 0\right) & N_{>\kappa x}\left(-s_{1},-s_{0}\right)=0 ; \\
+W_{-\operatorname{tag}}^{\varrho+\delta}(0, x) \geq 7 \epsilon x
\end{array}\right\} \\
& \leq \mathbb{P}\left\{\begin{array}{l}
N_{>\kappa x}\left(-s_{0}, x\right)=1 \\
W_{\leq \kappa x}^{\frac{1}{2}}\left(-s_{1},-s_{0}\right) \geq \epsilon x
\end{array}\right\}+\mathbb{P}\left\{W_{\leq \kappa x}^{\varrho+\delta}\left(-s_{0}, 0\right) \geq \epsilon x\right\} \\
& +\mathbb{P}\left\{W_{\leq \kappa x}^{\varrho+\delta}(0, x) \geq \epsilon x\right\},
\end{aligned}
$$

where the second step follows from the definition of $s_{0}$. Each of the three terms can now be controlled by applying Lemma A.2, thus completing the proof.

Lemma 4.6 For $\delta, \epsilon, \eta>0$ sufficiently small and $\varrho<\frac{1}{2}$, we have

$$
P_{22}(x)=o\left(\mathbb{P}\left\{B^{r}>x\right\}\right) \quad \text { as } x \rightarrow \infty .
$$

Proof Again, let $-y, y \in\left[s_{0}, s_{1}\right]$, be the arrival epoch of the tagged flow. The upper bound for $V_{2}(0)$ is similar to the bound for $V_{2}(0)$ given by (27), see Lemma 4.5. Specifically, it is first readily verified that (26) can be modified into

$$
2 V_{1}(-y)+V_{2}(-y) \leq 2 V_{1}\left(-s_{1}\right)+V_{2}\left(-s_{1}\right)+2 W_{-\operatorname{tag}}^{\frac{1}{2}}\left(-s_{1},-s_{0}\right) .
$$

Because of the PS discipline, we have $B_{2}(-y, 0) \geq B_{1}^{\text {tag }}(-y, 0)$. Combining the above with (5) and (4) yields

$$
\begin{aligned}
V_{2}(0) & =V_{2}(-y)+B_{1}^{-}(-y, 0)+B_{1}^{\mathrm{tag}}(-y, 0)-B_{2}(-y, 0) \\
& \leq V_{2}(-y)+V_{1}(-y)+A_{1}^{-}(-y, 0)-(\varrho+\delta) y+(\varrho+\delta) y-V_{1}^{-}(0) \\
& \leq V_{2}(-y)+V_{1}(-y)+W_{-\operatorname{tag}}^{\varrho+\delta}\left(-s_{1}, 0\right)+(\varrho+\delta) y-V_{1}^{-}(0) .
\end{aligned}
$$


We now distinguish between two different cases: (i) The large tagged flow already left before time 0 , and (ii) the large tagged flow is still present at time 0 .

First consider case (i). Using (4), it holds that

$$
\begin{aligned}
B_{1}(0, x) & \leq V_{1}^{-}(0)+A_{1}(0, x) \\
& \leq V_{1}^{-}(0)+W^{\varrho+\delta}(0, x)+(\varrho+\delta) x .
\end{aligned}
$$

Thus, using (29)-(31),

$$
\begin{aligned}
V_{2}(0)+B_{1}(0, x) \leq & 2 V_{1}\left(-s_{1}\right)+V_{2}\left(-s_{1}\right)+2 W_{-\operatorname{tag}}^{\frac{1}{2}}\left(-s_{1},-s_{0}\right) \\
& +W_{-\operatorname{tag}}^{\varrho+\delta}\left(-s_{1}, 0\right)+(\varrho+\delta) s_{1}+W^{\varrho+\delta}(0, x)+(\varrho+\delta) x \\
\leq & (\varrho+\delta+\eta) x+(\varrho+\delta) s_{1}+2 W_{-\operatorname{tag}}^{\frac{1}{2}}\left(-s_{1},-s_{0}\right) \\
& +\frac{3}{2} W_{-\operatorname{tag}}^{\varrho+\delta}\left(-s_{1}, 0\right)+W^{\varrho+\delta}(0, x) \\
\leq & \left(1-\frac{13}{2} \epsilon\right) x+2 W_{-\operatorname{tag}}^{\frac{1}{2}}\left(-s_{1},-s_{0}\right)+\frac{3}{2} W_{-\operatorname{tag}}^{\varrho+\delta}\left(-s_{1}, 0\right)+W^{\varrho+\delta}(0, x),
\end{aligned}
$$

where, in the second step, we added the term $\frac{1}{2} W_{-\operatorname{tag}}^{\varrho+\delta}\left(-s_{1}, 0\right)$ for consistency with case (ii) below and we used that $2 V_{1}\left(-s_{1}\right)+V_{2}\left(-s_{1}\right) \leq \eta x$, while the final step follows from the definition of $s_{1}$.

Next consider case (ii). Due to the PS discipline, we have $B_{1}^{\mathrm{tag}}(-y, 0)=B_{2}(-y, 0)$. Moreover, because the total service capacity is used during $[-y, 0]$, we obtain, using (3) and (4), that

$$
\begin{aligned}
B_{1}^{\mathrm{tag}}(-y, 0) & =\frac{1}{2}\left(y-B_{1}^{-}(-y, 0)\right) \\
& =\frac{1}{2}\left(y-V_{1}(-y)-A_{1}^{-}(-y, 0)+V_{1}^{-}(0)\right) .
\end{aligned}
$$

Note that $B_{1}^{\text {tag }}(0, x) \leq B_{\text {tag }}-B_{1}^{\text {tag }}(-y, 0)$. Combining the above and using (4) yields

$$
\begin{aligned}
B_{1}(0, x)= & B_{1}^{-}(0, x)+B_{1}^{\operatorname{tag}}(0, x) \\
\leq & V_{1}^{-}(0)+A_{1}(0, x)+B_{\operatorname{tag}}-\frac{1}{2}\left(y-V_{1}(-y)-A_{1}^{-}(-y, 0)+V_{1}^{-}(0)\right) \\
\leq & V_{1}^{-}(0)+W^{\varrho+\delta}(0, x)+(\varrho+\delta) x+B_{\operatorname{tag}} \\
& +\frac{1}{2} V_{1}(-y)+\frac{1}{2}\left(W_{-\operatorname{tag}}^{\varrho+\delta}\left(-s_{1}, 0\right)+(\varrho+\delta-1) y\right)
\end{aligned}
$$


Thus, applying (29), (30), and (32),

$$
\begin{aligned}
V_{2}(0)+B_{1}(0, x) \leq & 2 V_{1}\left(-s_{1}\right)+V_{2}\left(-s_{1}\right)+2 W_{-\operatorname{tag}}^{\frac{1}{2}}\left(-s_{1},-s_{0}\right)+\frac{3}{2} W_{-\operatorname{tag}}^{\varrho+\delta}\left(-s_{1}, 0\right) \\
& +W^{\varrho+\delta}(0, x)+(\varrho+\delta) x+B_{\operatorname{tag}}+\frac{1}{2}(3(\varrho+\delta) y-1) \\
\leq & \left(1-\frac{13}{2} \epsilon\right) x+2 W_{-\operatorname{tag}}^{\frac{1}{2}}\left(-s_{1},-s_{0}\right)+\frac{3}{2} W_{-\operatorname{tag}}^{\varrho+\delta}\left(-s_{1}, 0\right) \\
& +W^{\varrho+\delta}(0, x),
\end{aligned}
$$

where the final step follows from $2 V_{1}\left(-s_{1}\right)+V_{2}\left(-s_{1}\right) \leq \eta x$ and the upper bound of the service requirement of the tagged flow $B_{\mathrm{tag}}$.

Combining the sample-path relations for the cases (i) and (ii) with (19) provides the following bound:

$$
\begin{aligned}
& P_{22}(x) \\
& \quad \leq \mathbb{P}\left\{\begin{array}{c|l}
\left(1-\frac{13}{2} \epsilon\right) x+2 W_{-\operatorname{tag}}^{\frac{1}{2}}\left(-s_{1},-s_{0}\right) & N_{>\kappa x}\left(-s_{1},-s_{0}\right)=1 ; \\
+\frac{3}{2} W_{-\operatorname{tag}}^{\varrho+\delta}\left(-s_{1}, 0\right)+W^{\varrho+\delta}(0, x) \leq(1-2 \epsilon) x & N_{>\kappa x}\left(-s_{0}, x\right)=0
\end{array}\right\} \\
& \leq \mathbb{P}\left\{W_{\leq \kappa x}^{\frac{1}{2}}\left(-s_{1},-s_{0}\right) \geq \epsilon x\right\}+\mathbb{P}\left\{W_{\leq \kappa x}^{\varrho+\delta}\left(-s_{1}, 0\right) \geq \epsilon x\right\}+\mathbb{P}\left\{W_{\leq \kappa x}^{\varrho+\delta}(0, x) \geq \epsilon x\right\} .
\end{aligned}
$$

Again, each of the three above terms can be controlled by an application of Lemma A.2, which completes the proof.

Lemma 4.7 For $0<\delta<\frac{1}{2}-\varrho, 0<\epsilon<(1-\varrho-\delta) / 5$, and $\varrho<\frac{1}{2}$, we have

$$
P_{1}(x)=o\left(\mathbb{P}\left\{B^{r}>x\right\}\right) \quad \text { as } x \rightarrow \infty .
$$

Proof Using (4) and (5) in addition to the fact that the total service capacity is used during $\left[-s^{\star}, 0\right]$, we have

$$
\begin{aligned}
V_{1}(0)+V_{2}(0)= & V_{1}\left(-s^{\star}\right)+V_{2}\left(-s^{\star}\right)+A_{1}\left(-s^{\star}, 0\right)+B_{1}\left(-s^{\star}, 0\right)-s^{\star} \\
= & 2 V_{1}\left(-s^{\star}\right)+V_{2}\left(-s^{\star}\right)+2 A_{1}\left(-s^{\star}, 0\right)-s^{\star}-V_{1}(0) \\
= & 2 V_{1}\left(-s^{\star}\right)+V_{2}\left(-s^{\star}\right)+2\left(A_{1}\left(-s^{\star}, 0\right)-(\varrho+\delta) s^{\star}\right) \\
& -(1-2(\varrho+\delta)) s^{\star}-V_{1}(0) \\
\leq & \max \left\{2 V_{1}\left(-s_{1}\right)+V_{2}\left(-s_{1}\right)-(1-2(\varrho+\delta)) s_{1}, 0\right\} \\
& +2 W^{\varrho+\delta}\left(-s_{1}, 0\right)-V_{1}(0)
\end{aligned}
$$

where the last step follows by distinguishing between $s^{\star}=s_{1}$ and $s^{\star}<s_{1}$ (in addition to $\delta \leq \frac{1}{2}-\varrho$ ). Using (4) once more yields

$$
B_{1}(0, x) \leq V_{1}(0)+A_{1}(0, x) .
$$


Combining the above and using the definitions (20) and (21), we obtain

$$
\begin{aligned}
V_{2}(0)+B_{1}(0, x) \leq & \max \left\{2 V_{1}\left(-s_{1}\right)+V_{2}\left(-s_{1}\right)-(1-2(\varrho+\delta)) s_{1}, 0\right\} \\
& +2 W^{\varrho+\delta}\left(-s_{1}, 0\right)+W^{\varrho+\delta}(0, x)+(\varrho+\delta) x .
\end{aligned}
$$

Using (19), this sample-path relation can now be converted into a probabilistic bound:

$$
\begin{aligned}
P_{1}(x) \leq & \mathbb{P}\left\{\begin{array}{c}
\max \left\{2 V_{1}\left(-s_{1}\right)+V_{2}\left(-s_{1}\right)-(1-2(\varrho+\delta)) s_{1}, 0\right\}+2 W^{\varrho+\delta}\left(-s_{1}, 0\right) \\
+W^{\varrho+\delta}(0, x)+(\varrho+\delta) x \geq x(1-2 \epsilon) \\
2 V_{1}\left(-s_{1}\right)+V_{2}\left(-s_{1}\right) \leq(1-\varrho-\delta-5 \epsilon) x+(1-2(\varrho+\delta)) s_{1} \\
N_{>\kappa x}\left(-s_{1}, x\right)=0
\end{array}\right\} \\
\leq & \mathbb{P}\left\{2 W^{\varrho+\delta}\left(-s_{1}, 0\right)+W^{\varrho+\delta}(0, x) \geq 3 \epsilon x \mid N_{>\kappa x}\left(-s_{1}, x\right)=0\right\} \\
\leq & \mathbb{P}\left\{W_{\leq \kappa x}^{\varrho+\delta}\left(-s_{1}, 0\right) \geq \epsilon x\right\}+\mathbb{P}\left\{W_{\leq \kappa x}^{\varrho+\delta}(0, x) \geq \epsilon x\right\}
\end{aligned}
$$

where we used that $\epsilon \leq(1-\varrho-\delta) / 5$ in the second step. Both terms can be controlled by Lemma A.2. This completes the proof.

Lemma 4.8 For all $\eta, \kappa>0$ sufficiently small, $\kappa>0$ and $\varrho<\frac{1}{2}$, we have

$$
P_{3}(x)=o\left(\mathbb{P}\left\{B^{r}>x\right\}\right) \quad \text { as } x \rightarrow \infty .
$$

Proof This probability corresponds to the combination of two unlikely events. Specifically, since $2 V\left(-s_{1}\right)+V_{2}\left(-s_{1}\right)$ and $N_{>\kappa x}\left(-s_{1}, x\right)$ are independent, we have

$$
P_{3}(x) \leq \mathbb{P}\left\{2 V\left(-s_{1}\right)+V_{2}\left(-s_{1}\right)>\eta x\right\} \mathbb{P}\left\{N_{>\kappa x}\left(-s_{1}, x\right)=1\right\} .
$$

It follows from Theorem 2.1 and Lemma A.1 that $P_{3}(x)$ is bounded by $o\left(\mathbb{P}\left\{B^{r}>x\right\}\right)$ as $x \rightarrow \infty$.

Lemma 4.9 For any $\kappa>0$, we have

$$
P_{4}(x)=o\left(\mathbb{P}\left\{B^{r}>x\right\}\right) \quad \text { as } x \rightarrow \infty .
$$

Proof This follows directly from Lemma A.1.

\section{Conclusion and discussion}

The main conclusion of our paper is that if $B$ is regularly varying of index $v$, then so is the flow transfer delay; the steady-state workload, sojourn time, and queueing delay, however, are regularly varying of index $1-v$. The results for the flow transfer delay and workload followed in a rather straightforward fashion from earlier results; 
the derivation of the asymptotics of the sojourn time and queueing delay turned out to be substantially more involved. The proof relies on the following principles: (1) First a most likely scenario is identified; (2) a lower bound follows from computing the asymptotics corresponding to the most likely scenario; (3) then it is shown that all other scenarios provide negligible contributions compared to the most likely scenario.

It is interesting to compare the sojourn-time asymptotics of this system with those of corresponding FCFS and PS systems. Under FCFS a sojourn time is extremely long essentially because the job under consideration finds an extremely long queue, and this long queue is the result of one of the previous jobs being long. This explains why the tail of the sojourn time resembles the tail of the workload, which (Pakes 1975) is known to behave as the tail of $B^{r}$, i.e., regularly varying of index $1-v$. Under PS, on the contrary, the sojourn time is long because the job itself is large, and therefore the tail behaves as the tail of $B$, i.e., regularly varying of index $-v$. The heuristics behind the sojourn-time asymptotics of our model reveal that the sojourn time is large mainly due to finding a long queue, thus explaining why the corresponding tail probability vanishes as a regularly varying function of index $1-v$. However, in addition to a long queue, the long flow also affects the sojourn time in this system by reducing the service capacity of the buffer. These effects lead to interesting most likely scenarios for a large sojourn time to occur.

In the model we considered in this paper the queue is allocated the same share of the service capacity as each of the transmitting flows. It could be expected that such a policy may lead to relatively large buffer content of the queue. Alternatively, one may decide to assign a higher weight to the queue than to the flows; one could for instance serve the queue at rate $2 /(n+2)$ when there are $n$ flows present. Under such a policy multiple extremely large jobs are needed to cause a long sojourn time. This will be reflected in the corresponding asymptotics, cf. Zwart et al. (2004).

Open Access This article is distributed under the terms of the Creative Commons Attribution Noncommercial License which permits any noncommercial use, distribution, and reproduction in any medium, provided the original author(s) and source are credited.

\section{Appendix A: Technical lemmas}

Here we present two technical lemma's that can be frequently encountered in studies of queues with regularly varying service times, and that are used in Sect. 4.2. For proofs, we refer to e.g. Bekker et al. (2005); Zwart et al. (2004).

Lemma A.1 For any $k \in \mathbb{N}, \kappa>0$, and $\gamma>0$,

$$
\mathbb{P}\left\{N_{>\kappa x}(-\gamma x, 0) \geq k\right\}=O\left(\mathbb{P}\left\{B^{r}>x\right\}^{k}\right), \quad \text { as } x \rightarrow \infty .
$$

Lemma A.2 There exists a $\kappa^{*}>0$ such that for all $\kappa \in\left(0, \kappa^{*}\right]$, as $x \rightarrow \infty$,

$$
\mathbb{P}\left\{\sup _{0 \leq s \leq \gamma x}\left\{A_{2}(-s, 0)-(\varrho+\delta) s\right\}>\epsilon x \mid N_{>\kappa x}(-\gamma x, 0)=0\right\}=o\left(\mathbb{P}\left\{B^{r}>x\right\}\right)
$$


The same holds for the time-reversed case, i.e., there exists a $\kappa^{*}>0$ such that for all $\kappa \in\left(0, \kappa^{*}\right]$, as $x \rightarrow \infty$,

$$
\mathbb{P}\left\{\sup _{0 \leq s \leq \gamma x}\left\{A_{2}(0, s)-(\varrho+\delta) s\right\}>\epsilon x \mid N_{>\kappa x}(0, \gamma x)=0\right\}=o\left(\mathbb{P}\left\{B^{r}>x\right\}\right),
$$

\section{References}

Bekker R, Borst SC, Núñez-Queija R (2005) Performance of TCP-friendly streaming sessions in the presence of heavy-tailed elastic flows. Perf Eval 61:143-162

Bingham NH, Goldie CM, Teugels JL (1987) Regular variation. Cambridge University Press, Cambridge

Boxma OJ, Foss SG, Lasgouttes J-M, Núñez-Queija R (2003) Waiting time asymptotics in the single server queue with service in random order. Queueing Systems 46:35-73

Cohen JW (1973) Some results on regular variation for distributions in queueing and fluctuation theory. J Appl Probab 10:343-353

Crovella M, Bestavros A (1996) Self-similarity in world wide web traffic: evidence and possible causes. In: Proceedings of the ACM Sigmetrics '96, pp 160-169

Guillemin F, Robert Ph, Zwart AP (2003) Tail asymptotics for processor sharing queues. Adv Appl Probab 36:525-543

Mandjes MRH, Roijers F (2007) A fluid system with coupled input and output, and its application to bottlenecks in ad hoc networks. Queueing Systems 56:79-92

Pakes AG (1975) On the tails of waiting-time distributions. J Appl Probab 12:555-564

Reddy TB, Karthigeyan I, Manoj BS, Murthy CSR (2006) Quality-of-service provisioning in ad hoc wireless networks: a survey of issues and solutions. Ad Hoc Networks 4:83-124

Reich E (1958) On the integrodifferential equation of Takács. I. Ann Math Stat 29:563-570

Roijers F, Van den Berg JL, Mandjes MRH (2008) Performance analysis of differentiated resource-sharing in a wireless ad hoc network. Report PNA-E0807, CWI, The Netherlands

Van den Berg JL, Mandjes MRH, Roijers F (2006) Performance modeling of a bottleneck node in an IEEE 802.11 ad hoc network. In: Kunz T, Ravi SS (eds) Ad hoc now 2006. In: Fifth international conference on ad hoc networks and wireless "ad hoc now", Ottawa. Lecture Notes in Computer Science (LNCS) Series, vol 4104, pp 321-336

Zwart AP, Borst SC, Mandjes MRH (2004) Exact asymptotics for fluid queues fed by multiple heavy-tailed on-off flows. Ann Appl Probab 14:903-957

Zwart AP, Boxma OJ (2000) Sojourn time asymptotics in the M/G/1 processor sharing queue. Queueing Systems 35:141-166 